\begin{tabular}{|c|l|}
\hline Title & $\begin{array}{l}\text { Molecular dynamics study on evaporation and reflection of monatomic molecules to construct kinetic boundary } \\
\text { condition in vapor-liquid equilibria }\end{array}$ \\
\hline Author(s) & Kobay ashi, Kazumichi; Hori, Kazumasa; Kon, Misaki; Sasaki, Kiy ofumi; W atanabe, Masao \\
\hline Citation & $\begin{array}{l}\text { Heat and Mass Transfer, 52(9), 1851-1859 } \\
\text { https://doi.org/10.1007/300231-015-1700-6 }\end{array}$ \\
\hline Issue Date & 2016-09 \\
\hline Doc URL & http://hdl.handle.net/2115/67078 \\
\hline Rights & The final publication is available at link.springer.com \\
\hline Type & article (author version) \\
\hline File Information & kobay ashi_HA MT-2015.pdf \\
\hline
\end{tabular}

Instructions for use 


\title{
Molecular dynamics study on evaporation and reflection of monatomic molecules to construct kinetic boundary condition in vapor-liquid equilibria
}

\author{
Kazumichi Kobayashi · Kazumasa Hori · Misaki Kon · Kiyofumi Sasaki · Masao \\ Watanabe
}

Received: date / Accepted: date

\begin{abstract}
Using molecular dynamics simulations, the present study investigates the precise characteristics of evaporating and reflecting monatomic molecules (argon) composing a kinetic boundary condition (KBC) in a vapor-liquid equilibria. We counted the evaporating and reflecting molecules utilizing two boundaries (vapor and liquid boundaries) proposed by the previous studies (Meland et al., in Phys Fluids 16:223-243, 2004, and Gu et al., in Fluid Phase Equilibria $297: 77-89,2010)$. In the present study, we improved the method using the two boundaries incorporating the concept of the spontaneously evaporating molecular mass flux. The present method allows us to count the evaporating and reflecting molecules easily, to investigate the detail motion of the evaporating and reflecting molecules, and also to evaluate the velocity distribution function of the $\mathrm{KBC}$ at the vapor-liquid interface, appropriately. From the results, we confirm that the evaporating and reflecting molecules in the normal direction to the interface have slightly faster and significantly slower average velocities than that of the Maxwell distribution at the liquid temperature, respectively. Also, the stall time of the reflecting molecules at the interphase that is the region in the vicinity of the vapor-liquid interface is much shorter than those of the evaporating molecules. Furthermore, we discuss our method for constructing the KBC that incorporates condensation and evaporation coefficients. Based on these results, we suggest that the proposed method
\end{abstract}

Kazumichi Kobayashi, Kazumasa Hori, Misaki Kon, Kiyofumi Sasaki, Masao Watanabe

Dvision of Mechanical and Space Engineering

Hokkaido University, Kita 13 Nishi 8, Kita-ku, Sapporo, Hokkaido 060-8628, Japan

Tel.: +81-11-706-6429

Correspondence to: Kazumichi Kobayashi

E-mail: kobakazu@eng.hokudai.ac.jp is appropriate for investigating $\mathrm{KBC}$ in various nonequilibrium states or multi-component systems.

\section{Introduction}

With progression of advanced technologies using net evaporation/condensation $[1,2]$, heat and mass transfer between liquid and vapor phases has become very important in recent years from the viewpoints of molecular and micro-scale mass transfer. Net evaporation and condensation originate from molecules outgoing from and colliding onto a vaporliquid interface. Molecular gas dynamics (MGD) based on the molecular velocity distribution function, which is governed by the Boltzmann equation, is capable of describing vapor flows in the vicinity of the interface. In the analysis of MGD, the kinetic boundary condition (KBC) at the vaporliquid interface for the Boltzmann equation plays an essential role in the region near the interface [3-6]. The key physical processes for molecules at the interface are evaporation and reflection, because $\mathrm{KBC}$ is composed of molecules outgoing from the interface entering the vapor phase. KBC in a vapor-liquid equilibrium state or a weak nonequilibrium state (under a weak net condensation or evaporation) is written as follows $[7,8]$ :

$$
\begin{array}{r}
f_{\text {out }}=\rho_{\text {out }} \hat{f}_{\text {out }}^{*}=\frac{\rho_{\text {out }}}{\left(\sqrt{2 \pi R T_{\ell}}\right)^{3}} \exp \left(-\frac{\xi_{x}^{2}+\xi_{y}^{2}+\xi_{z}^{2}}{2 R T_{\ell}}\right), \\
\text { for } \xi_{z}>0,
\end{array}
$$

where $\rho_{\text {out }}$ in Eq. (1) is the vapor density composed of outgoing molecules from the interface entering the vapor phase, $\hat{f}_{\text {out }}^{*}$ is the normalized Maxwell distribution at liquid temperature $T_{\ell}, R$ is the gas constant, and $\xi_{x}, \xi_{y}$, and $\xi_{z}$ are the molecular velocities along the $x-, y-$, and $z$-physical axes, where $z$ is the direction normal to the interface and $x$ and $y$ are tangential to the interface. The positive $\xi_{z}$ is the molecular velocity from the interface to the vapor phase. 
In weak nonequilibrium states, $\rho_{\text {out }}$ is written as $\rho_{\text {out }}=$ $\alpha_{\mathrm{e}} \rho^{*}+\left(1-\alpha_{\mathrm{c}}\right) \sigma[7,8]$, where $\rho^{*}\left(T_{\ell}\right)$ is the saturated vapor density, $\alpha_{\mathrm{e}}$ and $\alpha_{\mathrm{c}}$ are the evaporation and condensation coefficients, respectively, and $\sigma$ is the parameter having the unit of density. The definitions of $\alpha_{\mathrm{e}}, \alpha_{\mathrm{c}}$, and $\sigma$ are discussed in Sec.3.4. In contrast, in vapor-liquid equilibrium states, $\rho_{\text {out }}$ becomes the saturated vapor density.

Equation (1) is determined from the evaporation and reflection of molecules at the interface. Hence, using the velocity distribution functions of the evaporating and reflecting molecules, $f_{\text {evap }}$ and $f_{\text {ref }}$, Eq. (1) can be rewritten as

$f_{\text {out }}=f_{\text {evap }}+f_{\text {ref }}=\rho_{\text {evap }} \hat{f}_{\text {evap }}+\rho_{\text {ref }} \hat{f}_{\text {ref }}, \quad$ for $\xi_{z}>0$,

where $\rho_{\text {evap }}$ and $\rho_{\text {ref }}$ are the densities of the vapor composed of evaporation and reflection molecules, respectively, and $\hat{f}_{\text {evap }}$ and $\hat{f}_{\text {ref }}$ are the normalized velocity distribution functions of the evaporating and reflecting molecules, respectively.

Several studies have been performed to determine the rate of evaporation and reflection of molecules based on molecular dynamics (MD) simulations (see, for example, Refs. [9-20]). As one of the methods to avoid the ambiguities introduced in the process of assigning evaporating or reflecting molecules based on MD simulations, a concept of spontaneous evaporation was assumed [12], and the molecular mass flux due to spontaneous evaporation was confirmed with MD simulation based on virtual-vacuum evaporation $[10,12]$. According to this assumption, the mass flux of spontaneously evaporating molecules depends only on the liquid temperature.

Here, the presence of spontaneously evaporating molecules in various equilibrium or nonequilibrium states is just an assumption; whether spontaneous evaporation of molecules in vapor-liquid equilibrium and nonequilibrium states actually occurs is not yet to be observed. Also, for binary mixtures, it is difficult to demonstrate spontaneous evaporation because it is not easy to maintain a constant mass concentration in a solution with virtual-vacuum evaporation, and the value of the evaporation coefficient is ambiguous (see, for example, Ref. [21]). Furthermore, for KBC, the velocity distribution function or the related molecular mass fluxes must be evaluated in the bulk vapor phase in the vicinity of the bulk liquid phase (defined below). However, in previous MD simulations, there were ambiguities for the positions of evaluating the velocity distribution function or related molecular mass fluxes. Thus, an alternative method should be urgently developed to obtain the evaporating molecular mass flux without using virtual-vacuum simulations and to understand the detailed behavior of evaporating and reflecting molecules related $\mathrm{KBC}$ in various equilibrium or nonequilibrium states.

Hence, in this study, we carefully investigate the characteristics of evaporation and reflection of molecules related to $\mathrm{KBC}$ using a method for counting the evaporating and reflecting molecules in the vapor-liquid equilibria. This method

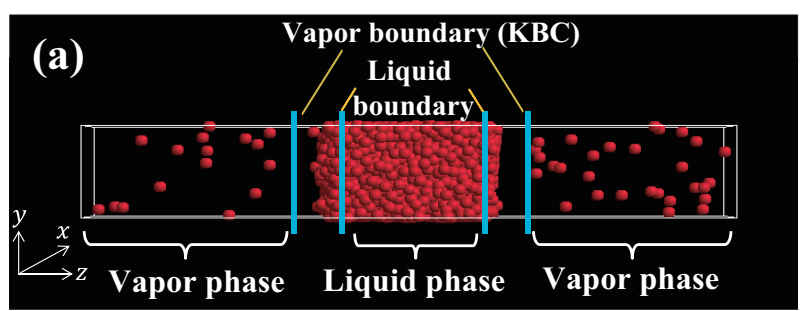

(b)

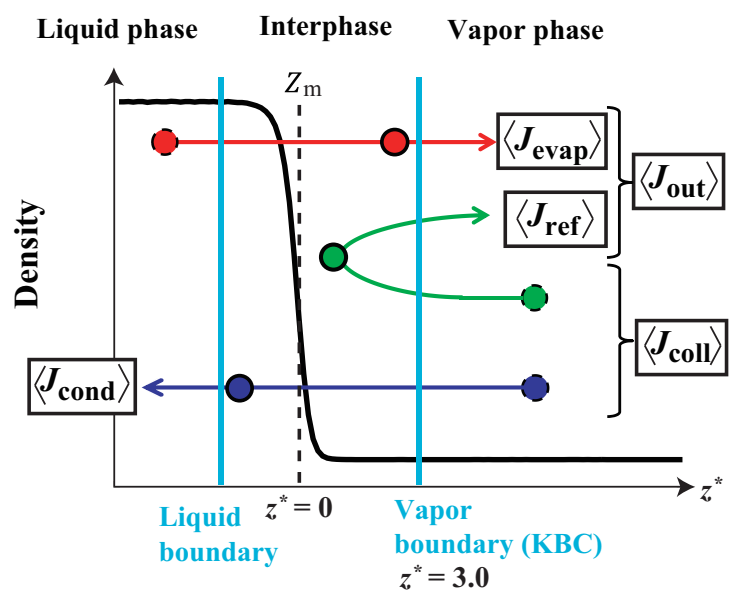

Fig. 1 (a) Snapshot of the MD simulation system at initial condition Red spheres denote argon molecules. (b) Schematic of the vapor-liquid interface. The red circles denote the evaporating molecules, the green circles denote the reflecting molecules, and the blue circles denote the condensation molecules.

uses the vapor and liquid boundaries as shown in Fig. 1. The concept of using two boundaries was proposed by Meland et al. [13] and Gu et al. [14]. We improved the method incorporating the concept of the spontaneously evaporating molecular mass flux. Furthermore, the construction method of $\mathrm{KBC}$, which includes the evaporation and condensation coefficients, as shown in Eq. (1), is discussed using the results of the present MD simulation.

\section{Methods}

\subsection{Equilibrium simulation}

In this section, we describe a method for vapor-liquid equilibrium MD simulations for argon molecules. A system composed of 4000 molecules was considered in a simulation box with dimensions $L_{x} \times L_{y} \times L_{z}$ at a temperature $T=85 \mathrm{~K}$ (the low temperature case for argon molecules in a vaporliquid equilibrium state), where $L_{x}=5 \mathrm{~nm}, L_{y}=5 \mathrm{~nm}$, and $L_{z}=33 \mathrm{~nm}$ (see Fig. 1(a)). Around the center of the $z$ direction $\left(L_{z}=16.5 \mathrm{~nm}\right)$, a plane liquid film is formed. Periodic boundary conditions were imposed on the simulation system ends in all three directions. 
For the intermolecular potential of argon molecules at $85 \mathrm{~K}$, we used a 12-6 type Lennard-Jones potential:

$\phi(r)=4 \varepsilon\left[\left(\frac{a}{r}\right)^{12}-\left(\frac{a}{r}\right)^{6}\right]$,

where the particle diameter $a$ is $3.405 \AA$, the potential depth $\varepsilon / k$ is $119.8 \mathrm{~K}$, and $k$ is the Boltzmann constant. Newton's equations of motion for the molecules in the system were solved by the leap-frog method. The cutoff radius was set to 15 Åin accordance with the previous studies [6,12], although it may be too short not to consider specific longrange interactions taking the extreme inhomogeneity of the two-phase system into account [22].

The initial condition was obtained from the temperaturecontrol simulation [23] of the system. After the system reached the vapor-liquid equilibrium state, we performed the equilibrium simulation without temperature control. The time increment was 5 fs, and the sampling period was 1 ps (200 time steps). To obtain the precise values of the macroscopic variables and the velocity distribution function, the simulation continued until $200 \mathrm{~ns}$. The densities of vapor and liquid obtained from the present simulation are $5.0 \mathrm{~kg} / \mathrm{m}^{3}$ and $1390 \mathrm{~kg} / \mathrm{m}^{3}$, which agree with the experimental ones [24]. As shown in Fig. 1(a), there are two vapor-liquid interfaces. Hence, we analyze the molecular behavior by replicating the simulation results of the left half of the simulation box as shown in Fig. 1(b).

\subsection{Counting of molecules in the interphase}

As shown in Fig. 1 (b), the bulk vapor and liquid phases contain the vapor and liquid boundaries, respectively. The region between the two boundaries is called the interphase. The concept of using two boundaries was discussed by Meland et al. [13] and Gu et al. [14]. This method allows the counting of evaporating and reflecting molecules at the vapor boundary. The definitions of both boundaries are important for counting molecular mass fluxes in MD simulations (Fig. 1 (b)). The evaporating molecular mass flux, $\left\langle J_{\text {evap }}\right\rangle$, was computed from the number of molecules crossing from the liquid boundary to the vapor boundary, where \langle\rangle denotes the time average. Conversely, the condensation molecular mass flux, $\left\langle J_{\text {cond }}\right\rangle$, was computed from the number of molecules crossing from the vapor boundary to the liquid boundary. The reflecting molecular mass flux, $\left\langle J_{\text {ref }}\right\rangle$, was computed from the number of molecules crossing the vapor boundary twice without crossing the liquid boundary as shown in Fig. 1 (b). These molecular mass fluxes were computed as

$$
\langle J\rangle=\frac{1}{n_{\mathrm{s}}} \sum_{n_{\mathrm{s}}} \frac{m \Delta N}{S \Delta t},
$$

where $n_{\mathrm{s}}$ is the sampling number, $\Delta N$ is the number of each molecule crossing the boundary during the time $\Delta t, m$ is the mass of a molecule, and $S$ is the cross-section area of the boundary. The outgoing molecular mass flux, $\left\langle J_{\text {out }}\right\rangle$, and colliding molecular mass flux, $\left\langle J_{\text {coll }}\right\rangle$, are computed as $\left\langle J_{\text {out }}\right\rangle=$ $\left\langle J_{\text {evap }}\right\rangle+\left\langle J_{\text {ref }}\right\rangle$ and $\left\langle J_{\text {coll }}\right\rangle=\left\langle J_{\text {cond }}\right\rangle+\left\langle J_{\text {ref }}\right\rangle$, respectively. We use the term colliding in accordance with the previous studies $[7,12,13]$. In this study, the colliding molecules mean the molecules having the velocities from the vapor phase to the liquid phase at the vapor boundary.

To utilize the above counting method, the positions of the vapor and liquid boundaries are important. Meland et al. [13] used the Soave-Redlich-Kwong (SRK) equation of state to determine the vapor boundary, as well as the geometrical method to determine the liquid boundary. Gu et al. [14] evaluated the boundaries using a spherical volume of interacting molecules from their MD simulation. In the present study, we determined the positions of the boundaries as follows.

First, we determined the position of the vapor boundary on the basis of our recent results [6,7]. This position was considered as the position of the KBC for the Boltzmann equation. Here, we introduced the new coordinate [12]:

$z^{*}=\frac{z-Z_{\mathrm{m}}}{\delta}$,

where $\delta$ is the 10-90 thickness of the density transition layer (in this simulation, $\delta$ became $6.33 \AA$ ). The center position, $Z_{\mathrm{m}}$, of the density transition layers is shown in Fig. 1 (b). Based on the previous studies [6,7], the vapor boundary position was defined in the range of $2.0<z^{*}<4.0$. Thus, we used $z^{*}=3.0$ in this simulation.

Next, the position of the liquid boundary was determined using the value of the spontaneously evaporating molecular mass flux with the virtual-vacuum evaporation simulation [25]. The details are discussed in Sec. 3.1. In the limit of vacuum evaporation, $\left\langle J_{\text {evap }}\right\rangle$ obtained from the present method takes the same value as the spontaneously evaporating molecular mass flux.

\section{Results}

\subsection{Evaporating molecular mass flux at the vapor boundary}

First, we confirmed that the obtained molecular mass fluxes reached the steady equilibrium state, thereby eliminating the influence of the initial condition of the molecules after the temperature-control simulation. Figure 2 shows (a) the time evolution of the number of molecules initially in the interphase, and (b) the time evolution of the evaporating molecular mass flux. As an example, we show the result for the positions of the vapor and liquid boundaries set at $z^{*}=3.0$ and $z^{*}=-3.0$, respectively. The evaporating molecular mass flux was the time averaged value over $1 \mathrm{~ns}$. 


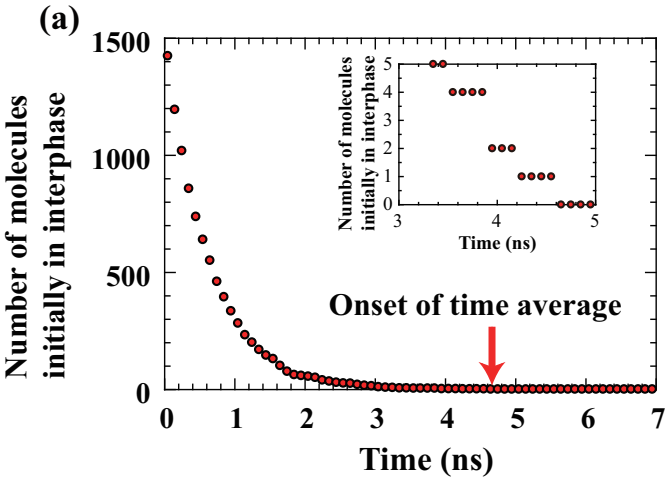

(b)

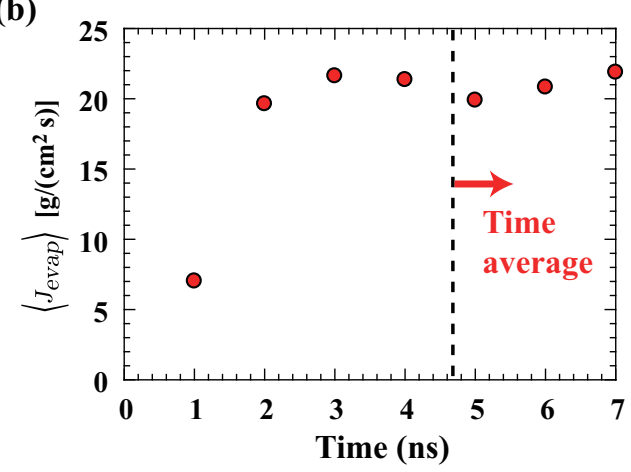

Fig. 2 Elimination of the initial condition. (a) Time evolution of number of molecules initially in the interphase for a vapor-liquid equilibrium state. (b) Time evolution of evaporating molecular mass flux. Each result was obtained for the vapor and liquid boundaries at $z^{*}=3.0$ and $z^{*}=-3.0$, respectively.

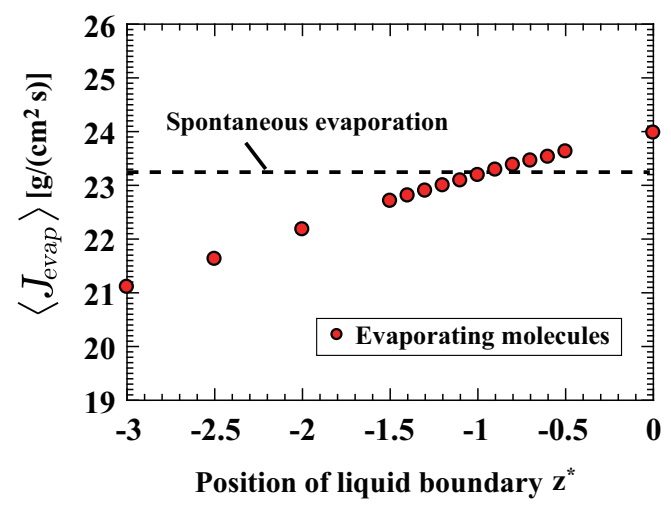

Fig. 3 Evaporating molecular mass flux at the vapor boundary. The red circles denote the evaporating molecules. The vapor boundary is at $z^{*}=3.0$.

Based on the result shown in Fig. 2(a), we confirmed that the number of molecules in the interphase at the initial condition became zero at $4.65 \mathrm{~ns}$ (see enlarged view of Fig. 2(a)). At this time, all molecules initially in the interphase moved out into the liquid or vapor phase. Furthermore, after $4.65 \mathrm{~ns}$, the evaporating molecular mass flux became approximately constant within statistical error [Fig. 2(b)] Thus, after $4.65 \mathrm{~ns}$, the time-averaged values of the molec- ular mass fluxes were taken. For the other cases where the liquid boundary was set to a different position, the same procedure was adopted.

Figure 3 shows the evaporating molecular mass flux as a function of the position of the liquid boundary. The dashed line is the spontaneously evaporating molecular mass flux under the virtual-vacuum simulation [25]. The position of the vapor boundary was set to $z^{*}=3.0$ in all cases. For the evaporating molecules in the equilibrium state, as the position of the liquid boundary moved toward the bulk vapor phase, the value gradually increased to that of $\left\langle J_{\text {out }}\right\rangle(=26.41$ $\mathrm{g} / \mathrm{cm}^{2} \mathrm{~s}$ ) at $T_{\ell}=85 \mathrm{~K}$ (when the liquid boundary reached the position of the vapor boundary, $\left\langle J_{\text {evap }}\right\rangle$ became $\left\langle J_{\text {out }}\right\rangle$ by the definition of this method). Here, once the position of the liquid boundary is specified, the value of $\left\langle J_{\text {evap }}\right\rangle$ is determined as shown in Fig. 3. The value of $\left\langle J_{\text {ref }}\right\rangle$ also changes with the position of liquid boundary. However, it should be emphasized that $\left\langle J_{\text {out }}\right\rangle$ still keeps the constant value regardless of the position of the liquid boundary ( $\left\langle J_{\text {out }}\right\rangle$ is obtained only from the vapor boundary).

Furthermore, from Fig. 3, when the position of the liquid boundary was $z^{*} \simeq-1.0$, the spontaneously evaporating and evaporating molecular mass fluxes were equal to each other. Hence, in this study, we determined the position of the liquid boundary at $z^{*}=-0.9$ in accordance with the value of spontaneously evaporating molecular mass flux. Hereafter, we investigated the molecular behavior at the vapor boundary $z^{*}=3.0$ with the liquid boundary set at $z^{*}=-0.9$.

\subsection{Evaporating and condensing molecules at the vapor boundary}

In the vapor-liquid equilibrium state, the evaporating and condensing molecular mass fluxes are evenly balanced. Thus, we investigated the characteristics of these molecules at the vapor boundary [Fig. 4(a)].

Figure 4(b) shows the normalized velocity distribution functions in the $z$ direction for evaporating and condensing molecules. In the present method, the velocity distribution function was measured by the surface perpendicular to the $z$ axis; evaporating molecules only had positive velocities in the $z$ direction, and the result was shown as a function of $\xi_{z} \hat{f}_{\text {evap }}^{z}$, where $\hat{f}_{\text {evap }}^{z}$ is the normalized velocity distribution function of evaporating molecules in the $z$ direction. On the other hand, the condensing molecules had negative velocities, as shown in Fig. 4(a). Thus, to facilitate comparison, we denoted the absolute value of the velocity of the condensing molecules as $\left|\xi_{z}\right|$ so that the measured velocity distribution function became $\left|\xi_{z}\right| \hat{f}_{\text {cond }}^{z}\left(\left|\xi_{z}\right|\right)$, where $\hat{f}_{\text {cond }}^{z}$ is the normalized velocity distribution function of condensing molecules in the $z$ direction. The abscissa of Fig. 4(b) is the molecular velocity normalized as $\zeta_{z}=\xi_{z} / \sqrt{2 R T_{\ell}}$. The total sampling number of evaporating molecules, $N_{\text {evap }}$, is 35046. 
(a)

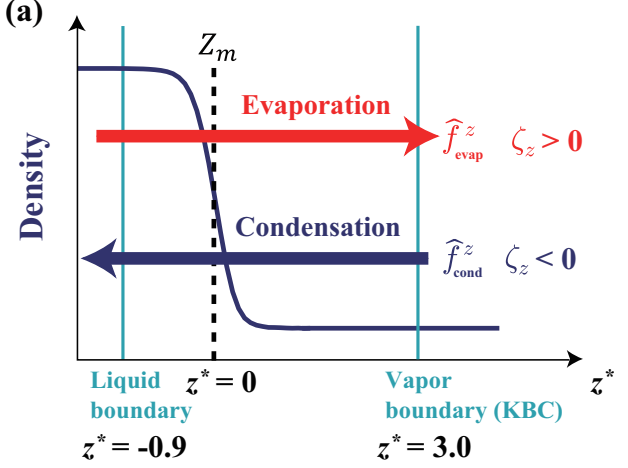

(b)

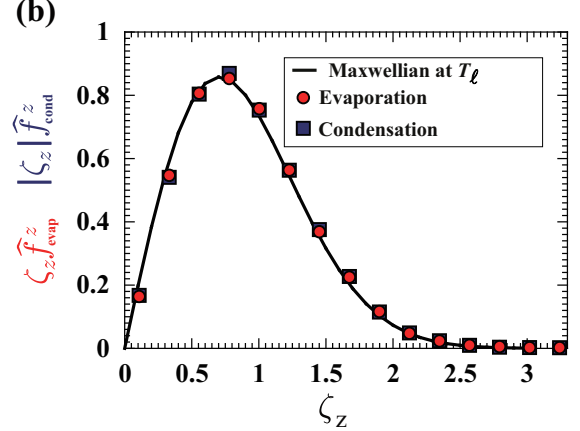

(9)

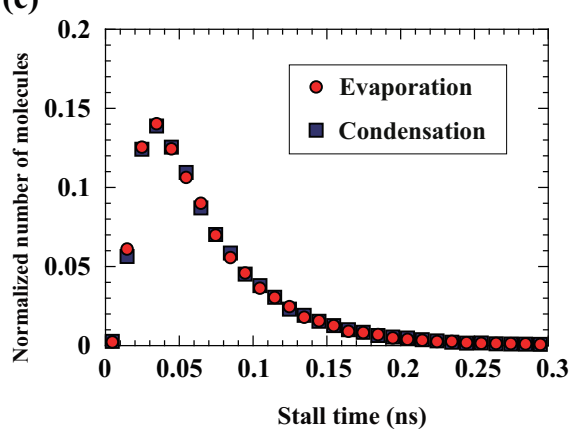

Fig. 4 Comparison of the evaporating and condensing molecules at the vapor boundary $z^{*}=3.0$. (a) Schematic of the definition of evaporating and condensing molecules. (b) Normalized velocity distribution function of evaporating and condensing molecules in the $z$ direction. (c) Stall time of evaporating and condensing molecules in the interphase between the liquid and vapor boundaries. The total sampling numbers of evaporating and condensing molecules are $N_{\text {evap }}=35046$ and $N_{\text {cond }}=35002$.

This sampling number is approximately the same as that of the condensing molecules, $N_{\text {cond }}=35002$.

From Fig. 4(b), we can see that the normalized velocity distribution functions of the evaporating and condensing molecules coincide with each other. Moreover, the velocity distribution functions of the evaporating and condensing molecules were almost the same as the Maxwell distribution at a liquid temperature of $85 \mathrm{~K}$. However, the temperatures of the velocity distribution functions were slightly higher than that of the Maxwell distribution: a temperature of approximately $89.5 \mathrm{~K}$ was obtained from the nonlinear least square method, which leads to the increase of average

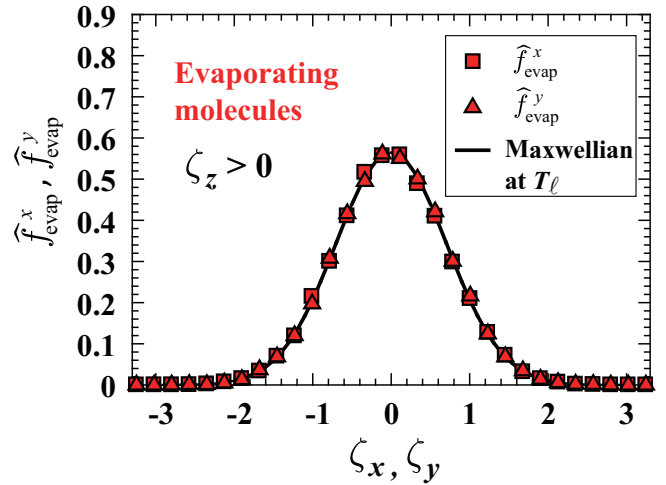

Fig. 5 Normalized velocity distribution functions in the $x$ and $y$ directions for evaporating molecules at the vapor boundary $z^{*}=3.0$.

velocity of evaporating or condensing molecules. According to the recent studies [16-18], when molecules evaporate or condense at the interface, the translational energy of the molecules becomes higher than that of molecules in the bulk phase. Also, the increasing of the temperature obtained from the velocity distribution function of evaporating molecules was reported $[11,18]$, which agrees with the present results.

To elucidate the characteristic behaviors of molecules, we investigated the stall time, which is the time for which evaporating and condensing molecules remained in the interphase between the liquid and vapor boundaries (Fig. 4(c)). The abscissa is the stall time and the ordinate is the numbers of evaporating and reflecting molecules normalized by $N_{\text {evap }}$ and $N_{\text {cond }}$, respectively. As shown in Fig. 4(c), the evaporating and condensing molecules have the maximum number at $0.04 \mathrm{~ns}$ and the tendency of these molecules coincide with each other. Consequently, we can confirm that the system attained a vapor-liquid equilibrium state using the present method.

Figure 5 shows the normalized velocity distribution function of the evaporating molecules in the $x$ and $y$ directions at the vapor boundary, where $\hat{f}_{\text {evap }}^{x}$ and $\hat{f}_{\text {evap }}^{y}$ are the velocity distribution functions of the evaporating molecules in the $x$ and $y$ directions, respectively. From this figure, we see that these velocity distribution agreed with the normalized Maxwell distribution at the liquid temperature of $85 \mathrm{~K}$. This result takes the same tendency of the spontaneously evaporating molecules obtained in the previous studies [12,26]. Hence, hereafter, we will discuss the velocity distribution function in the $z$ direction normal to the boundary.

\subsection{Reflecting molecules at the vapor boundary}

We considered two normalized velocity distribution functions of reflecting molecules in the $z$ direction [Fig. 6(a)]: $\hat{f}_{\text {ref }}^{z+}$ is the normalized velocity distribution function of reflecting molecules after reflection from the interphase, and 
(a)

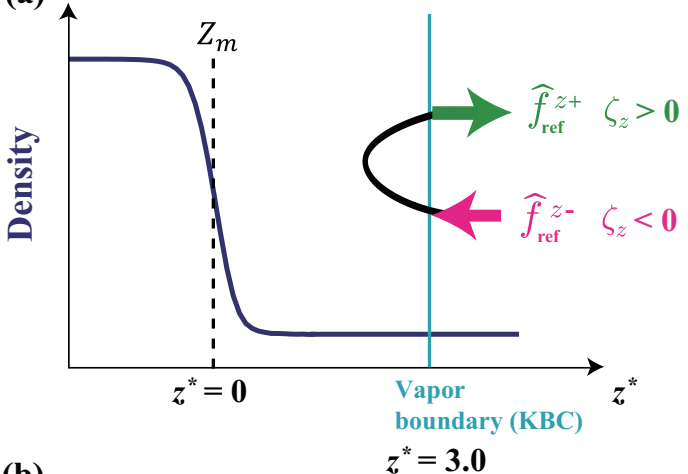

(b)

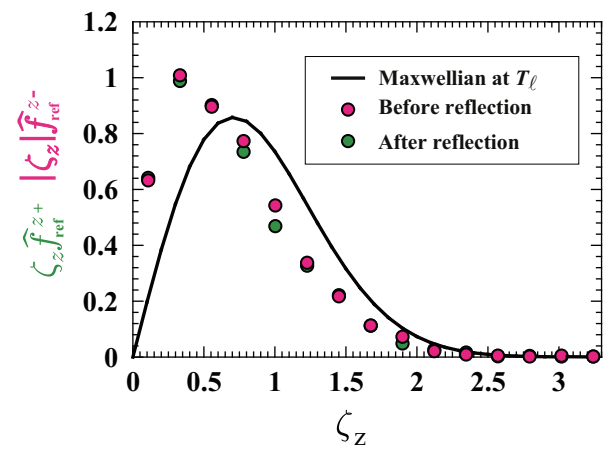

Fig. 6 Reflecting molecules at the vapor boundary $z^{*}=3.0$. (a) Schematic of the definition of velocity distribution functions of reflecting molecules. (b) Velocity distribution functions in the $z$ direction for reflecting molecules. The total sampling number of reflecting molecules, $N_{\text {ref }}$, is 4702 .

$\hat{f}_{\text {ref }}^{z-}$ is that of molecules before reflection from the interphase, where $\hat{f}_{\text {ref }}^{z-}$ is shown as an absolute velocity to facilitate comparison $\left|\xi_{z}\right| \hat{f}_{\text {ref }}^{z-}\left(\left|\xi_{z}\right|\right)$. Moreover, the total sampling number of reflecting molecules, $N_{\text {ref }}$, was 4702 in the present equilibrium simulation.

Figure 6(b) shows the normalized velocity distribution function of reflecting molecules. From this figure, we can see that these velocity distributions have a lower velocity than the normalized Maxwell distribution at the liquid temperature (i.e., the decrease of average velocity of reflecting molecules). For $\hat{f}_{\text {ref }}^{z+}$, the results for velocity distribution functions obtained by other measurement methods showed a similar tendency, as shown in Refs. [11,18]. Furthermore, before and after the reflection of the molecules, we found that the velocity distribution functions in the $z$ direction almost coincide with each other.

To investigate molecular reflection in more detail, the stall time of reflecting molecules was measured. Figure 7(a) shows the stall times of reflecting and evaporating molecules. From this figure, we see that the number of reflecting molecules with shorter stall times exceeded the number of evaporating molecules with such stall times. The reflecting molecules with longer stall times may have been reflected by multiparticle collisions, as discussed in Ref. [11]. (a)
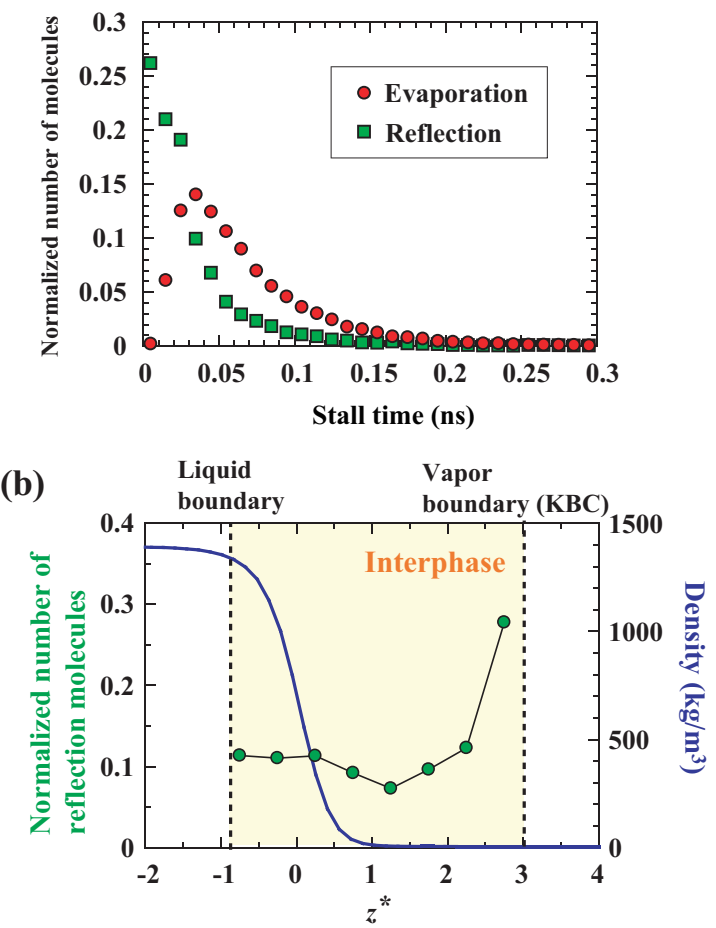

Fig. 7 Behaviors of reflecting molecules inside the interphase. (a) Stall time for reflecting and evaporating molecules inside the interphase between the liquid and vapor boundaries. (b) Reflection position of vapor molecules in the interphase. The total sampling number of reflecting molecules, $N_{\text {ref }}$, is 4702 .

From the perspective of MGD, the interaction time of the reflecting molecules (in this simulation, we define the interaction time as stall time of reflecting molecules) is negligibly small and molecular reflection is an instantaneous phenomenon $[3,4]$. The characteristic time is the mean free time of vapor molecules in MGD. In this simulation, the mean free time of the vapor was about $0.137 \mathrm{~ns}$, and the interaction time of almost all the reflecting molecules was smaller than the mean free time; $f_{\text {out }}$ composed of the reflecting molecules obtained from the present method was appropriate as the KBC of the Boltzmann equation.

We also investigated the reflection positions of molecules inside the interphase (Fig. 7(b)). The reflection position was defined as the maximum arrival position in the $z$-direction of reflection molecules in the interphase. The ordinate was normalized by the total number of reflecting molecules, $N_{\text {ref }}=$ 4702 , for each position. From this figure, we see that many reflecting molecules were reflected in the vicinity of the vapor boundary and more than $75 \%$ of the reflecting molecules were reflected in the range of $0.0<z^{*}<3.0$. Recently, it was reported that the velocity distribution of $f_{\text {out }}$ becomes anisotropic distribution during strong net condensation due to the incomplete accommodation between the liquid and reflecting molecules $[6,7,27]$. The reflection position and $\hat{f}_{\text {ref }}^{z-}$ 
relate to the accommodation process. Thus, the mechanism of the incomplete accommodation can be clarified by utilizing the present method for the nonequilibrium simulations.

\subsection{Kinetic boundary conditions and the evaporation and} condensation coefficients

Hereafter, we derived Eq. (1) from Eq. (2) using the definitions of the evaporation and condensation coefficients. As shown in the previous sections, we showed that $\hat{f}_{\text {evap }}^{z}$ and $\hat{f}_{\text {ref }}^{z+}$ deviate from the normalized Maxwell distribution at the liquid temperature. Some studies introduce the accommodation coefficient to discuss the deviation (for example, see Ref. [28]), and treat the KBC as a linear combination $f_{\text {evap }}$ and $f_{\text {ref. }}$. While it is one of the approaches, if $f_{\text {evap }}$ and $f_{\text {ref }}$ are combined into one as $f_{\text {out }}$, the analysis of the Boltzmann equation will be facilitated.

From Eq. (2), we could obtain the following mass flux relation using the definitions of the molecular mass fluxes:

$$
\begin{aligned}
\int_{\xi_{z}>0} \xi_{z} f_{\text {out }} \mathrm{d} \boldsymbol{\xi} & =\left\langle J_{\text {out }}\right\rangle, \int_{\xi_{z}>0} \xi_{z} f_{\text {evap }} \mathrm{d} \boldsymbol{\xi}=\left\langle J_{\text {evap }}\right\rangle \\
\int_{\xi_{z}>0} \xi_{z} f_{\text {ref }} \mathrm{d} \boldsymbol{\xi} & =\left\langle J_{\text {ref }}\right\rangle
\end{aligned}
$$

where $\int_{\xi_{z}>0} \mathrm{~d} \boldsymbol{\xi}$ denotes $\int_{0}^{\infty} \int_{-\infty}^{\infty} \int_{-\infty}^{\infty} \mathrm{d} \xi_{x} \mathrm{~d} \xi_{y} \mathrm{~d} \xi_{z}$. Hence

$\left\langle J_{\text {out }}\right\rangle=\left\langle J_{\text {evap }}\right\rangle+\left\langle J_{\text {ref }}\right\rangle$,

where $\left\langle J_{\text {out }}\right\rangle$ is written as $\left\langle J_{\text {out }}\right\rangle=\rho_{\text {out }} \sqrt{R T_{\ell} / 2 \pi}$ from Eq. (1). The collision molecular mass flux, $\left\langle J_{\text {coll }}\right\rangle$, is defined as follows [3]:

$-\int_{\xi_{z}<0} \xi_{z} f_{\text {coll }} \mathrm{d} \boldsymbol{\xi}=\sigma \sqrt{\frac{R T_{\ell}}{2 \pi}}=\left\langle J_{\text {coll }}\right\rangle$,

where $f_{\text {coll }}$ is the velocity distribution function of colliding molecues to the interface, and $\int_{\xi_{z}<0} \mathrm{~d} \boldsymbol{\xi}$ denotes $\int_{-\infty}^{0} \int_{-\infty}^{\infty} \int_{-\infty}^{\infty}$ $\mathrm{d} \xi_{x} \mathrm{~d} \xi_{y} \mathrm{~d} \xi_{z}$. The velocity distribution function of the whole molecular velocity space of vapor at the interface, $f$, is $f=$ $f_{\text {coll }}+f_{\text {out }}$. By using $\left\langle J_{\text {coll }}\right\rangle$, Eq. (6) is written as

$\rho_{\text {out }}=\frac{\left\langle J_{\text {evap }}\right\rangle}{\rho^{*} \sqrt{\frac{R T_{\ell}}{2 \pi}}} \rho^{*}+\frac{\left\langle J_{\text {ref }}\right\rangle}{\left\langle J_{\text {coll }}\right\rangle} \sigma$,

where evaporation coefficient, $\alpha_{\mathrm{e}}$, and condensation coeficient, $\alpha_{\mathrm{c}}$, are defined as $[6,12,7]$

$\alpha_{\mathrm{e}}=\frac{\left\langle J_{\text {evap }}\right\rangle}{\rho^{*} \sqrt{\frac{R T_{\ell}}{2 \pi}}}, \quad \alpha_{\mathrm{c}}=\frac{\left\langle J_{\text {cond }}\right\rangle}{\left\langle J_{\text {coll }}\right\rangle}=\frac{\left\langle J_{\text {coll }}\right\rangle-\left\langle J_{\text {ref }}\right\rangle}{\left\langle J_{\text {coll }}\right\rangle}$.

In this definition, $\alpha_{\mathrm{e}}$ is the function of only liquid temperature if $\left\langle J_{\text {evap }}\right\rangle$ is the spontaneously evaporating molecular mass flux and only depends on the liquid temperature. From Eqs. (8) and (9), we obtained the density in Eq. (1) as $\rho_{\text {out }}=\alpha_{\mathrm{e}} \rho^{*}+\left(1-\alpha_{\mathrm{c}}\right) \sigma$. Hence, we can derive Eq. (1) from Eq. (2). As shown above, the deviations of the velocity

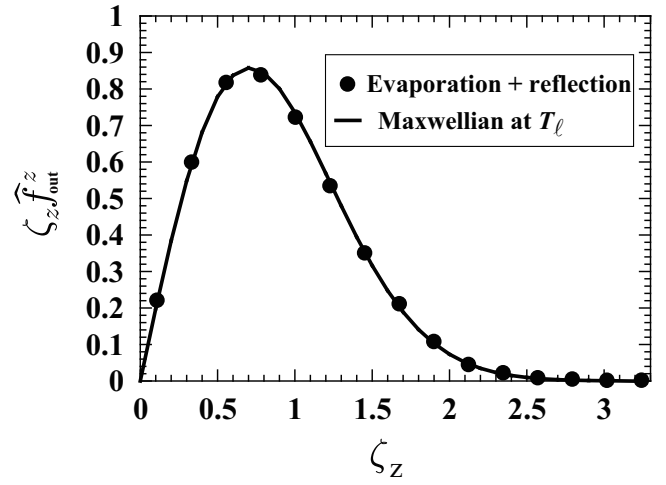

Fig. 8 Normalized velocity distribution function in the $z$ direction for outgoing molecules composed of evaporating and reflecting molecules at the vapor boundary $z^{*}=3.0$.

distribution functions, $\hat{f}_{\text {evap }}^{z}$ and $\hat{f}_{\text {ref }}^{z}$ (or $\hat{f}_{\text {cond }}^{z}$ ), from the normalized Maxwell distribution at the liquid temperature are included in the evaporation and condensation coefficients.

It is emphasized that we can construct the $\mathrm{KBC}$ of Eq. (1) knowing only $\left\langle J_{\text {evap }}\right\rangle,\left\langle J_{\text {cond }}\right\rangle$ (or $\left\langle J_{\text {ref }}\right\rangle$ ), and $\left\langle J_{\text {coll }}\right\rangle$ to determine the values of $\alpha_{\mathrm{e}}$ and $\alpha_{\mathrm{c}}$ in a vapor-liquid equilibrium or weak nonequilibrium state. As shown in Fig. 3, the values of $\left\langle J_{\text {evap }}\right\rangle$ and $\left\langle J_{\text {ref }}\right\rangle$ change depending on the position of the liquid boundary, which leads to the changes of the values of $\alpha_{\mathrm{e}}$ and $\alpha_{\mathrm{c}}$. However, $\left\langle J_{\text {out }}\right\rangle$ keeps the constant value and the functional form of Eq. (1) does not change. Hence, even if we choose the other position of liquid boundary, the correct values of mass, momentum, and energy fluxes are obtained from the $f_{\text {out }}$ constructed by the above procedure. Furthermore, if the vapor-liquid system is in the strong nonequilibrium state, the present procedure can be adopted when $\hat{f}_{\text {out }}^{z}$ is the normalized Maxwell distribution at the liquid temperature.

Here, as an example, we constructed $\hat{f}_{\text {out }}^{z}$ from $\hat{f}_{\text {evap }}^{z}$ and $\hat{f}_{\text {ref }}^{z+}$ of the present equilibrium simulation, where $\hat{f}_{\text {out }}^{z}$ is the normalized distribution function of the outgoing molecules in the $z$ direction, $\hat{f}_{\text {out }}^{z}=f_{\text {out }}^{z} / \rho^{*}$, where $\sigma$ becomes $\rho^{*}$ in the equilibrium state. Figure 8 shows the normalized velocity distribution function $\zeta_{z} \hat{f}_{\text {out }}^{z}$ constructed from $\zeta_{z} \hat{f}_{\text {evap }}^{z}$ and $\zeta_{z} \hat{f}_{\text {ref }}^{z+}$ according to

$\zeta_{z} \hat{f}_{\text {out }}^{z}=\alpha_{\mathrm{e}} \zeta_{z} \hat{f}_{\text {evap }}^{z}+\left(1-\alpha_{\mathrm{c}}\right) \zeta_{z} \hat{f}_{\text {ref }}^{z+}$,

where the value of the evaporation coefficient became the same as that of the condensation coefficient, $\alpha_{\mathrm{e}}=\alpha_{\mathrm{c}}=0.88$, in the present equilibrium MD simulation [25]. This value is the same as that obtained by a previous virtual-vacuum MD simulation [12]. From Fig. 8, $\zeta_{z} \hat{f}_{\text {out }}^{z}$ agreed with the Maxwell distribution at the liquid temperature. Hence, we constructed the Maxwell distribution, $\zeta_{z} \hat{f}_{\text {out }}^{z}$, from $\zeta_{z} \hat{f}_{\text {evap }}^{z}$ and $\zeta_{z} \hat{f}_{\text {ref }}^{z+}$.

In our previous study $[7,8]$, we showed that the velocity distribution of $f_{\text {out }}$ becomes the Maxwell distribution at the liquid temperature during weak net evaporation and con- 
densation, as shown in Eq. (1). Furthermore, the evaporation and condensation coefficients during net evaporation were obtained as

$\alpha_{\mathrm{e}}=\alpha_{\mathrm{c}}=\beta_{\mathrm{ne}}$,

and the evaporation and condensation coefficients during net condensation were obtained as

$\alpha_{\mathrm{e}}=\beta_{\mathrm{ne}}, \quad \alpha_{\mathrm{c}}=\frac{\rho^{*}}{\sigma}\left(\alpha_{\mathrm{e}}-\beta_{\mathrm{nc}}\right)+\beta_{\mathrm{nc}}$,

where $\beta_{\mathrm{ne}}$ and $\beta_{\mathrm{nc}}$ are constant parameters and the values for hard sphere molecules at liquid temperature $T_{\ell} / T_{c}=0.6$ were 0.871 and 0.928 , respectively, where $T_{c}$ is the critical temperature. From these results, we could see that the value of the condensation coefficient of hard-sphere monatomic molecules during net condensation increases with $\sigma$. On the contrary, for the polyatomic molecules (water and methanol), the value of the condensation coefficient decreases with $\sigma$ during net condensation in another one of our previous experimental studies [29].

Using the present procedure, the dependence of $\sigma$ on the condensation coefficient during net condensation can be clarified for various molecules from the view point of the molecular scale, and we can confirm whether spontaneously evaporating molecular mass flux depends only on liquid temperature in various nonequilibrium state. Furthermore, we can determine the $\mathrm{KBC}$ for binary mixture problems without using virtual-vacuum simulation [21].

\subsection{Comparison between the present and previous MD simulations}

We summarize the differences between the present and previous MD simulations.

As already mentioned above, the most important difference between the present and previous MD simulations is the evaluation position of the velocity distribution function of $\mathrm{KBC}$ at the vapor-liquid interface. For $\mathrm{KBC}$, the velocity distribution function must be evaluated in the bulk vapor phase in the vicinity of the bulk liquid phase [6,7]. However, in previous MD simulations [11-14,18], there were ambiguities in the positions of evaluating the velocity distribution function or related molecular mass fluxes. On the other hand, the position of KBC (vapor boundary position) was defined at $z^{*}=3.0$ in the present study, based on our previous studies $[6,7]$. According to the definition of the position of $\mathrm{KBC}$, we can investigate the velocity distribution function of evaporating and reflecting molecules and related molecular mass fluxes accurately.

We have discussed steady phase change in equilibrium state for only simple molecules (monatomic molecules) in the present study. Xie et al. [16,18], Cao et al. [17] and Louden et al. [19] investigated the phase change of polyatomic molecules (n-dodecane or water molecules). Gu et al. [15] studied unsteady nonequilibrium phase change using the multiscale molecular simulation. The present method can be adopted to the case of polyatomic molecules, unsteady nonequilibrium phase change, and higher liquid temperature. These studies are left as future works.

\section{Conclusions}

In the present study, we used MD simulation to investigate the evaporation and reflection of argon molecules in the vaporliquid equilibrium state at $85 \mathrm{~K}$. We counted the evaporating and reflecting molecules by improving the method of vapor and liquid boundaries proposed by the previous studies [13, 14] incorporating the concept of the spontaneously evaporating molecular mass flux. Furthermore, the present method enabled us to evaluate the velocity distribution function of $\mathrm{KBC}$ at the vapor-liquid interface, and allowed to count the evaporating and reflecting molecules in the interphase region between the liquid and vapor boundaries, investigating the motion of molecules in detail. From the present results, we confirmed that the average velocity of the evaporating molecules in the normal direction to the interface was slightly higher than that of the normalized Maxwell distribution at the liquid temperature. These results agree with the previous MD simulations $[11,18]$. We also found that the average velocities of the molecules before and after reflection were lower than that of the Maxwell distribution at the liquid temperature, and that the stall time of the reflecting molecules in the interphase was shorter than that of the evaporating molecules. Utilizing this method, we can construct $\mathrm{KBC}$ and analyze the detailed characteristics of evaporating and reflecting molecules in various vapor-liquid nonequilibrium states or multi-component systems.

\section{Compliance with Ethical Standards}

Conflict of Interest: The authors declare that they have no conflict of interest.

\section{References}

1. Lee J, Laoui T, Karnik R (2014) Nanofluidic transport governed by the liquid/vapour interface. Nature Nanotechnology 9:317-323. doi:10.1038/nnano.2014.28

2. Semiromi D Toghraie, Azimian AR (2012) Molecular dynamics simulation of annular flow boiling with the modified LennardJones potential function. Heat and Mass Transfer 48:141-152. doi:10.1007/s00231-011-0855-z

3. Sone Y (2007) Molecular Gas Dynamics: Theory, Techniques and Applications. Birkhäuser, .Boston

4. Cercignani C (2000) Rarefied Gas Dynamics: From Basic Concepts to Actual Calculations. Cambridge University Press, New York 
5. Frezzotti A (2011) Boundary conditions at the vapor-liquid interface. Phys. Fluids 23:030609. doi:10.1063/1.3567001

6. Fujikawa S, Yano T, Watanabe M (2011) Vapor-Liquid Interface, Bubble and Droplets: Fundamentals and Applications. SpringerVerlag, New York

7. Kon M, Kobayashi K, Watanabe M (2014) Method of determining kinetic boundary conditions in net evaporation/condensation. Phys. Fluids 26:072003. doi:10.1063/1.4890523

8. Kon M, Kobayashi K, Watanabe M (2014) Numerical analysis of kinetic boundary conditions at net evaporation/condensation interfaces in various liquid temperatures based on mean-field kinetic theory. AIP Conf. Proc. 1628:398-403. doi:10.1063/1.4902620

9. Matsumoto M (1998) Molecular dynamics of fluid phase change. Fluid Phase Equilibria 144:307-314. doi:10.1016/S03783812(97)00274-4

10. Anisimov SI, Dunikov DO, Zhakhovskii VV, Malyshenko SP (1999) Properties of a liquid-gas interface at high-rate evaporation. J. Chem. Phys. 110:8722-8729. doi:10.1063/1.478779

11. Tsuruta T, Tanaka H, Masuoka $\mathrm{T}$ (1999) Condensation/evaporation coefficient and velocity distributions at liquid-vapor interface. Int. J. Heat Mass Transfer 42:41074116. doi:10.1016/S0017-9310(99)00081-2

12. Ishiyama T, Yano T, Fujikawa S (2004) Molecular dynamics study of kinetic boundary condition at an interface between argon vapor and its condensed phase. Phys. Fluids 16:2899-2906. doi: $10.1063 / 1.1763936$

13. Meland R, Frezzotti A, Ytrehus T, Hafskjold B (2004) Nonequilibrium molecular-dynamics simulation of net evaporation and net condensation, and evaluation of the gas-kinetic boundary condition at the interphase. Phys. Fluids 16:223-243. doi:10.1063/1.1630797

14. Gu K, Watkins CB, Koplik J (2010) Molecular dynamics simulation of the equilibrium liquid-vapor interphase with solidification. Fluid Phase Equilibria 297:77-89. doi:10.1016/j.fluid.2010.06.014

15. Gu K,Watkins CB, Koplik J (2010) Multiscale molecular simulations of argon vapor condensation onto a cooled substrate with bulk flow. Phys. Fluids 22:112002. doi.org/10.1063/1.3517293

16. Xie JF, Sazhin SS, Cao BY (2011) Molecular dynamics study of the processes in the vicinity of the $n$-dodecane vapour/liquid interface. Phys. Fluids 23:112104. doi:10.1063/1.3662004

17. Cao BY, Xie JF, Sazhin SS (2011) Molecular dynamics study on evaporation and condensation of n-dodecane at liquid-vapor phase equilibria. J. Chem. Phys. 134:164309. doi:10.1063/1.3579457

18. Xie JF, Sazhin SS, Cao BY (2012) Molecular dynamics study of condensation/evaporation and velocity distribution of $n$-dodecane at liquid-vapor phase equilibria. J. Thermal and Sci. Tech. 7:1-13. doi:10.1299/jtst.7.x

19. Louden P, Schoenborn R, Lawrence CP (2013) Molecular dynamics simulations of the condensation coefficient of water. Fluid Phase Equilib. 349:83-86. doi:10.1016/j.fluid.2013.04.006

20. Kryukov AP, Levashov VY, Pavlyukevichb NV (2014) Condensation coefficient: definitions, estimations, modern experimental and calculation data. J. Eng. Phys. Thermophys 87:237-245. doi:10.1007/s10891-014-1006-4

21. Frezzotti A (2011) Non-equilibrium structure of the vaporliquid interface of a binary fluid. AIP Conf. Proc. 1333:161-167. doi:10.1063/1.3562642

22. Janeček $J$ (2006) Long range corrections in inhomogeneous simulations. J. Phys. Chem. B 110:6264-6269. doi:10.1021/jp056344z

23. Allen MP, Tildesley DJ (1987) Computer Simulation of Liquid. Oxford University Press, New York

24. Tegeler CH, Span R, and Wagner W (1999) A new equation of state for argon convering the fluid region for temperatures from the melting line to $700 \mathrm{~K}$ at pressures up to $1000 \mathrm{MPa}$. J. Phys. Chem. Ref. Data 28:779-850.
25. Kobayashi K, Hori K, Yaguchi H, Watanabe M (2014) Molecular dynamics simulation on evaporation molecules in a vapor-liquid equilibrium state. AIP Conf. Proc. 1628:404-410. doi:10.1063/1.4902621

26. Frezzotti A, Gibelli L, Lorenzani S (2005) Mean field kinetic theory description of evaporation of a fluid into vacuum. Phys. Fluids 17:012102. doi:10.1063/1.1824111

27. Ishiyama T, Fujikawa S, Kurz T, Lauterborn W (2013) Nonequilibrium kinetic boundary condition at the vaporliquid interface of argon. Phys. Rev. E 88:042406. doi: http://dx.doi.org/10.1103/PhysRevE.88.042406

28. Tsuruta T, Tokunaga A, G. Nagayama (2011) Molecular boundary conditions and accommodation coefficient on a nonequilibrium liquid surface. AIP Conf. Proc. 1333:859-864 doi:10.1063/1.3562753

29. Kobayashi K, Watanabe S, Yamano D, Yano T, Fujikawa S (2008) Condensation coefficient of water in a weak condensation state. Fluid Dyn. Res. 40:585-596. doi:10.1016/j.fluiddyn.2007.12.011 